\title{
La qualité de la gouvernance microfinancière dans les pays de l'UEMOA : construction d'un indice agrégé de gouvernance des IMF appliquée au cas du Bénin
}

Pascal Wélé I*

\begin{abstract}
Résumé - Ce papier pose le problème de la qualité de la gouvernance dans le secteur microfinancier de l'UEMOA et suggère la construction d'un indice agrégé permettant d'évaluer les institutions opérant dans ce secteur à partir de l'exemple du Bénin. En effet, à l'instar des travaux dédiés à la gouvernance des entreprises classiques, dont ceux de Louizi (2006) et de Marsal et Bouaiss (2007) figurent parmi les plus récents sur le secteur bancaire, la littérature microfinancière met en évidence des liens étroits entre la qualité de la gouvernance des IMF et leur performance (Rock et al., 2001 ; Labie, 2001 ; Drake et Rhyne, 2002 ; Mersland et Strøm, 2007). Or la qualité de la gouvernance s'inscrit de plus en plus au premier plan des défis majeurs qu'affrontent la plupart des IMF en marche vers la pérennité dans les pays de l'UEMOA. De plus, en dépit de la multiplicité des facettes que présentent les crises de gouvernance dans plusieurs institutions, l'analyse des indices agrégés issus des données du Bénin révèle une dégradation de la qualité de la gouvernance sur l'ensemble du secteur. Ce constat tend à amincir, toutes choses égales par ailleurs, les perspectives de viabilité qui s'avèrent pourtant légitimes en matière de microfinance.
\end{abstract}

Mots clés - microfinance, gouvernance, performance.

Classification JEL - G21, G30, L31, O55.

Abstract - In this paper we address the issue of governance quality in the microfinance sector in the Economic and Monetary Union of West Africa and try to build an aggregate index for measuring the sustainability of these institutions focusing on the Benin case study. Following the recent literature on corporate governance in the classic bank sector (Louizi, 2006; Marsal \& Bouaiss, 2007), some studies show evidence of a strong correlation between the governance quality of microfinance organizations and their performance (Rock et al., 1998; Labie, 2001; Drake \& Rhyne, 2002;

* Docteur en Sciences de Gestion, Université d'Abomey-Calavi. 
Mersland \& Strøm, 2007). And governance quality increasingly tends to become the biggest challenge faced by most microfinance organizations in EMUWA. Despite the many facets of governance quality, the analysis of the aggregate indexes built using data from Benin shows that governance quality in the whole sector has been deteriorated. All other things being equal, this conclusion tends to reduce the prospects of viability in the sector.

Key words - microfinance, governance, performance.

JEL Classification - G21, G30, L31, O55.

\section{INTRODUCTION}

Au bout de décennies d'expérience, la microfinance a révélé toute sa vitalité dans l'économie des pays en développement en général et de ceux de l'Union économique et monétaire ouest-africaine (UEMOA) en particulier. En effet, on y observe, pour le cas spécifique du Bénin, un dynamisme prononcé de l'offre, qui se traduit tant par la croissance du nombre d'opérateurs actifs dans le secteur ${ }^{1}$ que par l'augmentation remarquable des dépôts et des crédits octroyés. Outre cette croissance, on note la diversité des IMF et des modes d'intervention dont la mission " commune " consiste à offrir des services financiers aux populations exclues du système bancaire. La loi dite PARMEC ${ }^{2}$ qui régit le secteur dans I'UEMOA, complétée par les instructions de la Banque centrale des États de l'Afrique de l'Ouest (BCEAO), distingue trois grands types d'IMF. D'après les statistiques de la Cellule de supervision du secteur de la microfinance du Bénin (CSSFD), les institutions mutualistes et coopératives (IMC) viennent en tête sur les plans de la couverture du marché (84,6 \% de la clientèle) et de la collecte de l'épargne ( $80 \%$ des dépôts). Par contre, l'octroi de crédit est dominé par les institutions de crédit direct (ICD) qui détiennent 59,9\% de l'offre. Les ONG et les projets à volet microfinancier (ONG) quant à eux n'occupent qu'une très faible part de marché sur tous les plans.

Selon la loi PARMEC, seules les IMC agréées peuvent collecter l'épargne du public. Les ICD qui opèrent sur la base d'une convention ${ }^{3}$ renouvelable regroupent les associations et sociétés spécialisées dans la microfinance. Enfin, pour les ONG, l'activité de microfinance est menée à titre accessoire. Elle s'intègre généralement dans le dispositif d'accompagnement nécessaire pour l'atteinte des objectifs stratégiques de l'ONG ou du projet.

Le poids social, économique et financier que représente la microfinance au Bénin et les avancées considérables qu'elle a connues ces dernières années justifient l'intérêt des réflexions sur la qualité de la gouvernance au sein des institutions. Ce papier se concentre donc sur la problématique de l'efficacité de la gouvernance

1. Les études dénombrent 762 institutions de microfinance au Bénin, dont seulement 279 sont enregistrées par la Cellule de Supervision du Secteur de la Microfinance (CSSFD, 2008).

2. PARMEC : Projet d'appui à la réglementation des mutuelles d'épargne et de crédit.

3. Voir Arrêté n 045/MF/DC/MICROFIN du 7 juin 1999. 
des IMF du Bénin. II suggère la construction d'un indice agrégé pour analyser la qualité de la gouvernance, à partir d'un échantillon d'IMF du Bénin.

\section{INTÉRÊT DE LA QUESTION DE LA GOUVERNANCE EN MICROFINANCE}

D'apparition récente en microfinance, la gouvernance constitue aujourd'hui l'un des thèmes centraux des débats portant sur la pérennité des IMF. Dans le contexte spécifique aux pays de I'UEMOA, ce constat s'explique, en partie, par les crises multiples et profondes qu'affronte le secteur, dont la croissance des impayés et l'accumulation des pertes d'exploitation figurent parmi les premiers signes.

II existe un lien parfois direct entre les défis d'institutionnalisation et de pérennité qui se posent aux IMF et ceux liés à la qualité de leur gouvernance (CMEF ${ }^{4}$, 2006). En effet, l'étude de Mersland et Strøm (2007), qui portait sur un échantillon de 226 IMF dans 57 pays dont 5 de l'UEMOA (Bénin, Burkina Faso, Mali, Sénégal, Togo) a révélé un lien positif entre la qualité de la gouvernance et la performance des IMF mesurée par le ROA (Return on Assets). En d'autres termes, l'amélioration de la qualité de la gouvernance des IMF peut servir de levier pour accroître leur efficacité (Rock et al., 2001 ; Labie, 2001). Ces résultats confirment ceux obtenus par des études portant sur les firmes bancaires, qui ont également montré que les mécanismes de gouvernance exercent une influence positive sur la performance (Louizi, 2006 ; Marsal et Bouaiss ; 2007). Toutefois, une étude portant sur un échantillon plus réduit d'IMF d'Europe centrale et orientale a obtenu des résultats plus mitigés (Hartarska, 2005).

Trois facteurs clés semblent justifier l'intérêt d'un dispositif de gouvernance en microfinance (Campion et Frankiewicz, 1999). D'une part, face à une croissance forte et souvent rapide de leur volume d'activités, les IMF adoptent des structures plus complexes et gèrent des capitaux de plus en plus importants. Ceci nécessite des efforts particuliers de la part des dirigeants qui doivent maintenir des standards de rendement élevés, et nécessite par conséquent l'implication plus accrue du conseil d'administration pour assurer une gestion efficace. D'autre part, ces IMF, dont une part croissante est réglementée, doivent relever les défis qui en découlent. Par exemple, la collecte de l'épargne a des exigences en termes de gestion auxquelles il faut obéir pour offrir des garanties suffisantes aux déposants (Boyé et al., 2006). Enfin, la conquête d'un marché devenu concurrentiel suppose non seulement une vision stratégique bien définie, mais aussi un regard attentif sur l'efficacité opérationnelle (Mersland et Strøm, 2007).

La gouvernance des IMF, comme des firmes classiques, se réfère aux mécanismes par lesquels les divers apporteurs de ressources - donateurs et investisseurs privés - peuvent s'assurer que ces dernières seront utilisées dans la stricte poursuite des objectifs qu'ils ont préalablement retenus (Clarkson et Deck, 1997 ; Labie, 2001). En effet, depuis la séparation des pouvoirs actionnarial et managérial (Berle et Means, 1932), le contrôle du management des dirigeants

4. CMEF : Council of Microfinance Equity Funds. 
ne manque pas d'intérêt dans la firme, les objectifs et buts poursuivis par les différentes parties prenantes pouvant diverger, voire s'opposer parfois. Parmi les problèmes de gouvernance qui varient suivant le type d'IMF, l'attention est souvent focalisée sur les conflits d'intérêts entre les " dirigeants-gestionnaires » et les " propriétaires » apporteurs de capitaux (Labie, 2005 ; Mersland et Strøm, 2007). D'après la littérature dédiée à la gouvernance d'entreprise, ces conflits peuvent induire des comportements opportunistes de la part des dirigeants et sont souvent inducteurs des coûts d'agence dont la minimisation est le but principal des mécanismes de gouvernance (Jensen et Meckling, 1976).

En microfinance, les problèmes d'agence deviennent croissants lorsqu'on intègre la double vocation des institutions. D'une part, elles doivent atteindre le maximum de clients parmi les couches les plus pauvres, en vue de contribuer notablement au développement des pays concernés. D'autre part, la première vocation doit être remplie sur des bases financièrement viables et pérennes. Or, si certains auteurs pensent que ces deux missions sont compatibles, d'autres par contre trouvent cette compatibilité bien plus complexe pour les IMF (Woller et al., 1999). Il est donc évident que, selon la configuration du dispositif de gouvernance mis en place, certains éléments produisent des effets distincts sur les chances de l'IMF de réaliser chacune des deux missions mesurées par l'accessibilité et la viabilité ${ }^{5}$.

Toutefois, selon Clarkson et Deck (1997), nombre d'IMF d'Afrique subsaharienne sont, historiquement, impulsées soit par les pouvoirs publics désireux de combattre le chômage et la pauvreté par la promotion du micro-entrepreneuriat, soit par des ONG soucieuses de maximiser l'impact de leur intervention à travers un dispositif additionnel axé sur l'offre des services financiers aux populations non bancarisées, et/ou de servir de relai d'un système financier défaillant. Ainsi, ces types d'IMF n'ont pas de propriétaires qui puissent exercer leurs droits au sens propre du terme (Hartarska, 2005). De même, dans la majorité des cas, malgré l'importance des ressources financières mobilisées par les IMF, les personnes impliquées dans les choix stratégiques, qu'il s'agisse du conseil d'administration (CA) ou de la direction, n'ont qu'un intérêt financier insignifiant dans l'institution. Or, au cœur du système de gouvernance des IMF se trouve le CA (Labie, 2005), dont le rôle de contrôle du management des dirigeants, pour être efficace, implique une réelle motivation de chacune des personnes qui le composent (Campion et Frankiewicz, 1999 ; Rock et al., 2001). Pour une gouvernance efficace, Jensen (1993) suggère, entre autres, la détention d'un nombre significatif de titres par le dirigeant et les membres du CA, pour qu'existe une certaine convergence d'intérêts avec les actionnaires. C'est pourquoi, au Bénin, dans la plupart des IMF de crédit direct, la direction générale joue un rôle déterminant dans le processus de décision du CA, depuis la phase de préparation jusqu'à la phase de mise en œuvre. Ce constat qui est confirmé par les évaluations de Planet Rating (2003,

5. Ce phénomène s'observe dans les CA, lorsque ceux-ci comprennent en leur sein des donateurs et des investisseurs privés. Les premiers tendent à se focaliser sur l'accessibilité tandis que les seconds privilégient la rentabilité. Ce faisant, cette relation conflictuelle peut se transposer sur la configuration du CA dont les membres sont représentants des donateurs comme des investisseurs privés (Hartarska, 2005, p. 1630). 
2005), en particulier pour PADME, PAPME et Vital Finance, montre l'intérêt des processus de transformation dans lesquels s'engagent certaines IMF du Bénin. Ceci permettrait de clarifier leur structure de propriété, en vue de la mise en place d'un système de gouvernance dont il faut espérer que l'efficacité contribue à stimuler la performance.

\section{UN OUTIL D'ANALYSE DE LA GOUVERNANCE DES IMF}

\subsection{L'efficacité et la structure du conseil d'administration}

Au regard de la multiplicité des défis de la gouvernance microfinancière dont les sources varient selon le type d'institution et la structure de propriété, on peut envisager qu'il existe une diversité de mécanismes de gouvernance. Néanmoins, l'attention est portée sur Oster (1995) qui met en évidence le rôle crucial du CA dans le cas spécifique des organisations d'économie sociale. En effet, certains mécanismes qui fonctionnent naturellement dans les entreprises commerciales peuvent être partiellement ou totalement inexistants dans le cadre des organisations du tiers-secteur (Labie, 2005). Dans ces conditions, le CA s'avère essentiel, car il constitue le tampon naturel entre les dirigeants-gestionnaires de l'organisation et les intérêts extérieurs.

Face à ce constat qui décrit la microfinance des pays en développement, certains analystes de la gouvernance se focalisent sur la manière dont le CA exerce son contrôle pour s'assurer que les dirigeants prennent des décisions favorables à la réalisation de sa double mission. Par exemple, Campion et Frankiewicz (1999) et Mersland et Strøm (2007) ont mis l'accent sur les variables telles que la taille du CA, sa composition, la rotation des principaux dirigeants et la durée de mandat des membres du CA, ainsi que la séparation des pouvoirs entre le directeur général et le président du CA. Pour les IMF cotées en bourse, le CMEF (2006) intègre les systèmes de rémunération des membres du CA. II faut néanmoins noter que ces variables ne constituent pas une liste exhaustive. De plus, les variables telles que la taille et la composition du CA, ainsi que le système de rémunération de ses membres impliquent que les unités observées soient homogènes; ce qui n'est pas le cas de notre échantillon. En effet, dans les cas où les IMF de l'échantillon sont hétérogènes, notamment du point de vue du statut juridique et de la structure organisationnelle, une comparaison axée sur ces variables perdrait en pertinence et exigerait des analyses au cas par cas.

\subsection{L'indice agrégé de gouvernance et ses variables}

Face à l'hétérogénéité des IMF de l'échantillon, il semble utile de se référer à des études menées par la Banque mondiale et le Fonds monétaire international qui 
ont comparé la qualité du management des organisations issues de plusieurs secteurs d'activités. Parmi ces études, le FMI (2004), Briceno-Garmendia et Foster (2007) et Mbangala (2007) suggèrent un indice agrégé de gouvernance qui regroupe une série de critères basés essentiellement sur des données binaires. En effet, cet indice permet de déterminer dans quelle mesure les unités observées sont gérées sur des bases commercialement viables. Ces critères couvrent plusieurs dimensions dont quatre requièrent une attention particulière, en raison des possibilités d'application qu'elles offrent aux entreprises de microfinance. II s'en dégage une batterie de neuf critères qui sont résumés dans le tableau 1.

Parmi les avantages de l'indice agrégé de gouvernance, il faut noter sa capacité à intégrer la diversité des dimensions de la gouvernance (Briceno-Garmendia et Foster, 2007). À cela, il faut ajouter sa simplicité de mise en œuvre sur le plan méthodologique; une simplicité notamment fondée sur son recours aux variables binaires. En effet, comme le soulignent Evrard et al. (2003), les variables binaires " constituent la forme la plus élémentaire à laquelle peut être réduite toute mesure ». Toutefois, il faut être bien conscient que derrière cette simplicité se cache le risque d'exclure des variables d'analyse pertinentes qui ne seraient pas binaires. En outre, si pour certaines variables de l'indice agrégé, un statut binaire semble être acceptable, pour plusieurs autres par contre, la réalité correspond nettement plus à des positions multiples constituant plutôt un continuum. C'est notamment le cas des variables telles que le respect des normes prudentielles, l'indépendance vis-à-vis des pouvoirs publics, la cohérence des manuels de procédures et l'autonomie du CA. Cette remarque montre l'intérêt de compléter l'analyse de l'indice de gouvernance ainsi définie, en intégrant des variables de gouvernance de type quantitatif.

Tableau 1 : Les variables de mesure de la qualité de gouvernance des IMF

\begin{tabular}{|l|l|l|}
\hline \multicolumn{1}{|c|}{ Variables } & \multicolumn{1}{|c|}{ Indicateurs } & \multicolumn{1}{c|}{ Modalités } \\
\hline $\begin{array}{l}\text { Respect de la } \\
\text { législation }\end{array}$ & $\begin{array}{l}\text { Autorisation d'exercice } \\
\text { Respect des normes prudentielles }\end{array}$ & $\begin{array}{l}\text { '1' si l'IMF est autorisée, '0' si non } \\
\text { '1' si } 100 \% \text { des règles prudentielles sont } \\
\text { respectes, '0' si non }\end{array}$ \\
\hline $\begin{array}{l}\text { Autonomie } \\
\text { managériale }\end{array}$ & $\begin{array}{l}\text { Vis-à-vis des pouvoirs publics } \\
\text { Vis-à-vis des bailleurs de fonds }\end{array}$ & $\begin{array}{l}\text { '1'si indépendance vis-à-vis des pouvoirs publics, } \\
\text { '0' si non } \\
\text { '0's'il y a influence des bailleurs, '1' si non }\end{array}$ \\
\hline $\begin{array}{l}\text { Qualité du } \\
\text { système } \\
\text { d'information }\end{array}$ & $\begin{array}{l}\text { Cohérence des manuels de procédures } \\
\text { Disponibilité des rapports d'audit } \\
\text { Participation à une évaluation } \\
\text { internationale }\end{array}$ & $\begin{array}{l}\text { '1's' s'il y a cohérence des manuels, '0' si non rapports d'audit sont disponibles, '0' si non } \\
\text { '1' si l'IMF participe à une évaluation internationale, } \\
\text { '0' si non }\end{array}$ \\
\hline $\begin{array}{l}\text { Conseil } \\
\text { d'Administration }\end{array}$ & $\begin{array}{l}\text { Séparation des fonctions de Directeur } \\
\text { Général et de Président du CA } \\
\text { Pouvoir de décision }\end{array}$ & $\begin{array}{l}\text { '1's'il y a séparation des pouvoirs, '0' si non } \\
\text { '1' si le CA est autonome, '0' si non }\end{array}$ \\
\hline
\end{tabular}

Source : Adapté de FMI (2004), Briceno-Garmendia et Foster (2007). 


\section{LA QUALITÉ DE LA GOUVERNANCE DES OPÉRATEURS BÉNINOIS DE MICROFINANCE : ANALYSE DES RÉSULTATS}

À partir la précédente description des variables, le tableau annexé (Annexe 1) présente l'indice agrégé de gouvernance qui caractérise les IMF du Bénin, notamment à travers ses quatre dimensions. D'après ce tableau, la moyenne de l'indice de gouvernance pour les 27 IMF que compte l'échantillon est de 73,3 \% ; ce qui signifie qu'en général, les IMF observées au Bénin répondent à un peu plus de six critères sur les neuf qu'exigerait une gouvernance efficace. Néanmoins, la distribution des IMF sur les classes d'indices indique une plus grande concentration des indices de gouvernance entre $60 \%$ et $70 \%$. C'est en effet la tendance qu'illustre le graphique 1 , selon lequel près de $30 \%$ des IMF se situent dans les limites comprises entre $60 \%$ et $70 \%$, limites qui définissent la classe modale de la qualité de gouvernance de l'échantillon. Par ailleurs, pour l'ensemble des IMF observées au Bénin, le principe de la séparation des pouvoirs entre le directeur général et le président du conseil d'administration s'est avéré bien respecté et ne fait l'objet d'aucun débat.

\section{Graphique 1 : Distribution des IMF sur les classes d'indices de gouvernance}

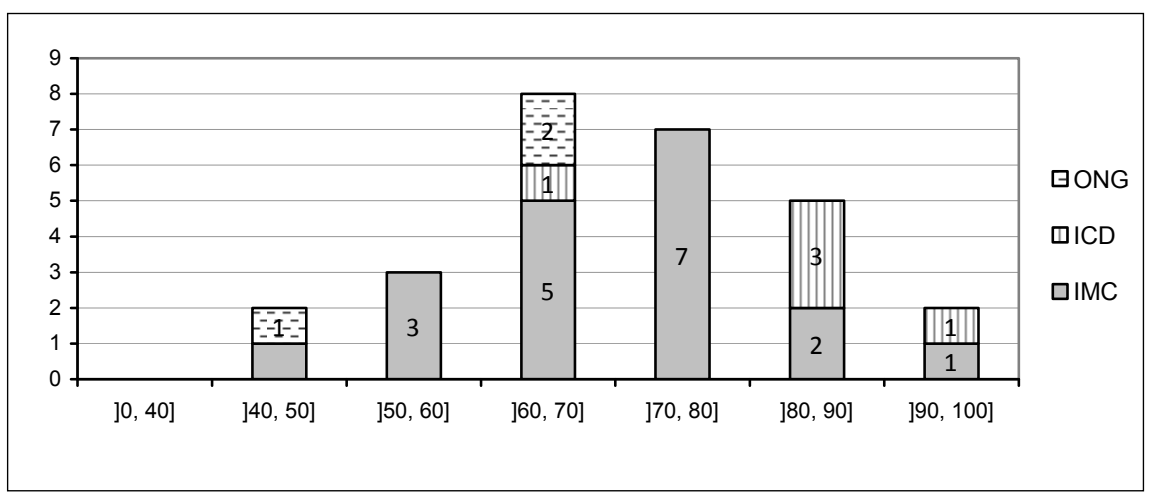

S'agissant des formes institutionnelles, le test de Kruskal-Wallis (Tableau 2) indique une différence significative au seuil de $5 \%$ entre la qualité de gouvernance des groupes d'IMF constitués à partir de leur forme juridique, à savoir les institutions mutualistes et coopératives (IMC), les institutions de crédit direct (ICD) et les $O N G$ et projets à volet microfinancier (ONG). II en est de même pour la taille du conseil d'administration qui diffère sensiblement suivant le type d'institution.

De ce tableau, il ressort que les ICD ont le système de gouvernance le plus efficace, puisqu'elles occupent le rang moyen le plus élevé dans le classement des indices par ordre croissant, avec un indice moyen de 86,7 \%. De plus, il faut noter que, dans ce groupe, PAD se distingue en étant la seule IMF de l'échantillon à réaliser un score de gouvernance de $100 \%$. 
Tableau 2 : Test de Kruskal-Wallis sur les indices de gouvernance

\begin{tabular}{|l|c|c|c|c|c|}
\hline \multirow{2}{*}{\multicolumn{1}{|c|}{ Variables }} & \multicolumn{3}{c|}{ Rangs moyens } & \multirow{2}{*}{ Khi-deux (2 ddl) ${ }^{6}$} & \multirow{2}{*}{ Seuil de significativité } \\
\cline { 2 - 4 } & IMC & ICD & ONG & & \\
\hline Nombre d'IMF & 19 & 5 & 3 & - & - \\
\hline Indice de gouvernance & 13,29 & 21,00 & 6,83 & $6,833^{*}$ & 0,033 \\
\hline Taille du CA & 17,37 & 3,90 & 9,50 & $14,940^{* *}$ & 0,001 \\
\hline
\end{tabular}

(*) Seuil de significativité de $5 \%$.

(**) Seuil de significativité de $1 \%$.

Source : Données collectées auprès des IMF, février 2007.

Par ailleurs, le tableau 3 qui évalue les modes d'intervention selon les critères de gouvernance précédemment retenus, permet de dresser les principaux défis propres à chaque groupe. Ainsi, pour trois ICD sur les cinq observées (soit 60 \%), des efforts s'avèrent encore nécessaires pour accroître l'autonomie décisionnelle du conseil d'administration. II faut signaler que seulement $60 \%$ des unités de ce groupe respectent les normes prudentielles, avec les risques de sanction que cela peut entraîner de la part de l'autorité de supervision.

Tableau 3 : La qualité de gouvernance des IMF par forme institutionnelle

\begin{tabular}{|l|l|c|c|c|c|}
\hline \multirow{2}{*}{ Dimensions } & \multicolumn{2}{|c|}{ Critères } & \multicolumn{3}{l}{ Part d'unités validées dans la catégorie (\%) } \\
\cline { 3 - 6 } & & IMC & ICD & ONG & Echantillon \\
\hline \multirow{2}{*}{$\begin{array}{l}\text { Respect de la } \\
\text { législation }\end{array}$} & Autorisation d'exercice & 89,5 & 100,0 & 66,7 & 88,9 \\
\cline { 2 - 6 } & Respect des normes prudentielles & 21,1 & 60,0 & 0,0 & 25,9 \\
\hline \multirow{2}{*}{$\begin{array}{l}\text { Autonomie } \\
\text { managériale }\end{array}$} & Autonomie vis-à-vis de l'État & 57,9 & 100,0 & 100,0 & 70,4 \\
\cline { 2 - 6 } & Autonomie vis-à-vis des bailleurs & 89,5 & 100,0 & 33,3 & 85,2 \\
\hline \multirow{2}{*}{$\begin{array}{l}\text { Qualité du système } \\
\text { d'information }\end{array}$} & Qualité des manuels de procédures & 84,2 & 100,0 & 100,0 & 88,9 \\
\cline { 2 - 6 } & Disponibilité des rapports d'audit & 78,9 & 100,0 & 33,3 & 63,0 \\
\cline { 2 - 6 } & Participation à une évaluation internationale & 57,9 & 80,0 & 66,7 & 63,0 \\
\hline \multirow{2}{*}{$\begin{array}{l}\text { Pouvoir du conseil } \\
\text { d'administration }\end{array}$} & Dualité entre direction et contrôle & 100,0 & 100,0 & 100,0 & 100,0 \\
\cline { 2 - 6 } & Autonomie décisionnelle & 63,2 & 40,0 & 33,33 & 55,6 \\
\hline
\end{tabular}

Source : Données collectées auprès des IMF, février 2007.

Les ICD sont suivies par les IMC dont l'indice moyen s'établit à 71,9\%, soit en dessous de la moyenne d'ensemble. La position de l'indice de gouvernance par rapport à la moyenne générale n'est qu'un reflet de l'inefficacité relative du système de gouvernance qui caractérise ce type d'institution. Parmi les défis auxquels les IMC sont confrontées, viennent en tête le respect des normes prudentielles (seulement $21 \%$ obéissent à cette règle) et l'autonomie managériale qui tend à s'effriter depuis quelques années, notamment avec l'intervention de l'État

6. Les valeurs de Khi-deux sont données avec deux degrés de liberté. 
dans les institutions en crise (seules $58 \%$ des IMC prennent des décisions sans l'intervention des pouvoirs publics). Pour illustrer ce constat, on peut évoquer le cas du réseau FECECAM qui est sous administration provisoire depuis 2006. Cette mesure fait suite à la dégradation continue des indicateurs de performance dont la qualité du portefeuille, et s'est traduite entre autres par la dissolution du CA du réseau. À ce défi relatif à l'autonomie managériale, il faut ajouter la qualité du système d'information et l'efficacité du pouvoir décisionnel du CA, qui constituent des défis non négligeables dans certaines IMF de ce groupe.

Il en est de même pour les ONG qui occupent le dernier rang en termes de qualité de gouvernance, avec un indice de 59,3\%. Or, en général, les ONG éprouvent de sérieuses difficultés sur le plan des performances financières. Cette tendance se confirme dans la mesure où aucune ONG n'est à la hauteur des normes prudentielles. En outre, les deux tiers des unités observées dans ce groupe subissent l'influence notable voire forte des partenaires financiers (en l'occurrence les donateurs) et/ou des fondateurs ${ }^{7}$ dans les choix stratégiques. Cette influence constitue un facteur défavorable à la qualité de la gouvernance, à l'instar de la qualité du système d'information et du pouvoir de décision et de contrôle du CA, qui mérite d'être renforcée pour deux tiers des IMF. Par ailleurs, si l'influence exercée par les partenaires sur les ONG explique la forte priorité accordée aux objectifs sociaux, elle pourrait néanmoins constituer, du moins à long terme, un frein au processus d'autonomisation du volet microfinancier de ces institutions. À partir de ces constats, on peut justifier certaines interrogations sur la capacité réelle des dispositifs de gouvernance mis en place à assurer l'autonomie organisationnelle nécessaire à la pérennité de ces IMF.

\section{CONCLUSION}

Le présent papier s'inscrit dans le courant de la littérature qui tend à considérer le mécanisme de gouvernance comme un levier pertinent pour améliorer la performance des entreprises qui opèrent dans la microfinance (Hartarska, 2005 ; Mersland et Strøm, 2007). Néanmoins, sa spécificité réside dans le fait qu'il suggère une évaluation de la qualité de gouvernance des IMF au travers d'un indice agrégé. De l'analyse des indices agrégés fournis par les données de l'échantillon tiré du secteur microfinancier du Bénin, il ressort que la qualité du dispositif de gouvernance varie de façon significative suivant la forme institutionnelle observée. Toutefois, on note une dégradation de la qualité de la gouvernance sur l'ensemble du secteur. Si les institutions de crédit direct semblent disposer de gouvernance plus efficace, les institutions mutualistes ou coopératives et les ONG et projets à volet microfinance doivent encore relever d'importants défis pour améliorer la qualité de leur gouvernance. Ceci relance, toutes choses égales par ailleurs, le débat sur les perspectives de viabilité et de pérennité qui s'offrent aux opérateurs de microfinance du Bénin.

7. C'est le cas notamment d'ID où l'influence du fondateur - ID France - est tangible : d'une part, le président et fondateur d'ID France siège dans le conseil d'administration d'ID au Bénin. D'autre part, le président de ce conseil d'administration est le directeur d'une ONG locale partenaire de I'ONG française. 


\section{BIBLIOGRAPHIE}

Berle A. A., Means G. C. (1932), "The Modern Cooperation and Private property", New York, Macmillan Company, $396 \mathrm{p}$.

Boye S., HajdenBerg J., Poursat C. (2006), « Le guide de la microfinance : microcrédit et épargne pour le développement », Paris, Éditions d’Organisation, Groupe Eyrolles, $304 \mathrm{p}$.

Bricenco-Garmendia, Foster (2007), "More Fiscal Resources for Infrastructure? Evidence from Est Africa", The World Bank, Sustainable Development Department, Africa Region, June, $89 \mathrm{p}$.

Campion A., Frankiewicz C. (1999), " Guide de la gouvernance efficace des institutions de microfinance », Document de Travail, $n^{\circ} 3$, The Microfinance Network, Washingon DC 2005, $101 \mathrm{p}$.

CharReAux G. (1997), « Le Gouvernement des entreprises : Corporate Gouvernance, théories et faits ", Paris, Economica, 540 p.

Clarkson M., DeCK M. (1997), "Effective Governance for Microfinance Institutions", in Churchill F.C. (1997), Establishing A Microfinance Industry : Governance, Best Practices, Access to Capital Markets, The Microfinance Network, Washington DC, 2005, p. 5-13.

Council of Microfinance Equity Funds (2006), "La pratique de gouvernance des institutions de microfinance cotées en bourse ", Exposé de consensus du Conseil de SICAV Microfinance, Council of Microfinance Equity Funds, janvier, 36 p.

JeNSEN M.C. (1993), "The Modern Industrial Revolution, Exit, and the Failure of Internal Control Systems", Journal of Finance, vol. 48, n³, juillet, p. 831-880.

Jensen M.C., MeCKLING W. H. (1976), "Theory of the firm: Managerial Behavior, Agency Cost, and Ownership Structure", Journal of Financial Economics, vol. $3 n^{\circ} 4$, p. 305-360.

LABIE M. (2005), « Comprendre et améliorer la gouvernance des organisations à but non lucratif : vers un apport des tableaux de bord ? ", Revue Gestion, vol. 30, n 1, Printemps, p. 78-86.

LABIE M. (2001), "Corporate governance in microfinance organizations: A long and winding road", Management Decision, vol. 39, p. 296-301.

LouIzI G. (2006), « Les mécanismes internes de gouvernance bancaire : importance et interactions ; application aux banques tunisiennes ", Working Paper, www.iecs. edu/congres/articles/article-final-louizi.pdf, $34 \mathrm{p}$.

MARSAL C., Boualss K. (2007), "Les mécanismes internes de gouvernance dans les banques : un état de l'art ", Notes de Recherche du FARGO - LEG, Pole d'Économie et de Gestion, Institut d'Administration des Entreprises de Dijon, 2 p.

Mbangala M. (2007), "Taking Stock of Railway Companies in Sub-saharan Africa", The World Bank Studies, Washington DC, November, 142 p.

Mersland R. et Strøm R. Ø. (2007), "Performance and Corporate Governance in Microfinance Institutions", MPRA Working paper, $n^{\circ}$ 3888, Agder University, Norway, June, Online at http://mpra.ub.uni-muenchen.de/3888/, 37 p.

Planet Rating (2005), « GIRAFE : PAPME Bénin », Rapport d'évaluation, juin, 20 p.

Planet Rating (2003), « GIRAFE : Vital Finance Bénin », Rapport d'évaluation, septembre, $39 \mathrm{p.}$

Planet Rating (2003), « GIRAFE : PADME Bénin », Rapport d'évaluation, mars, 40 p. 
Rock R., Otero M., Saltzman S. (2001), « Principes et pratiques de la gouvernance en microfinance », Microenterprise Best Practices, USAID, ACCION International, traduction française GRET, septembre, $52 \mathrm{p}$.

Woller G., Dunford C., WoOdWORTH W. (1999), "Where to Microfinance?”, International Journal of Economic Development, vol. 1, n 1, p. 29-64.

\section{ANNEXES}

Tableau : La qualité de la gouvernance des IMF du Bénin

\begin{tabular}{|c|c|c|c|c|c|c|c|c|c|c|}
\hline \multirow[b]{2}{*}{ IMF } & \multicolumn{2}{|c|}{$\begin{array}{l}\text { Respect de la } \\
\text { législation }\end{array}$} & \multicolumn{2}{|c|}{$\begin{array}{l}\text { Autonomie } \\
\text { managériale }\end{array}$} & \multicolumn{3}{|c|}{$\begin{array}{c}\text { Qualité du système } \\
\text { d'information }\end{array}$} & \multicolumn{2}{|c|}{ Contrôle du CA } & \multirow[b]{2}{*}{$\begin{array}{c}\text { Total } \\
(\%)\end{array}$} \\
\hline & 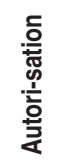 & 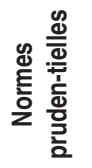 & 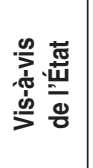 & 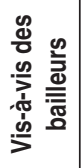 & 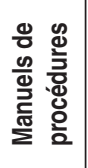 & 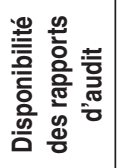 & 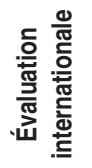 & 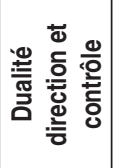 & 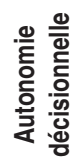 & \\
\hline \multicolumn{10}{|c|}{ Institutions Mutualistes ou Coopératives - IMC } & 71,9 \\
\hline $\mathrm{APH}$ & 0 & 1 & 1 & 0 & 0 & 0 & 0 & 1 & 0 & 44,4 \\
\hline $\mathrm{CBE}$ & 1 & 1 & 1 & 1 & 1 & 1 & 0 & 1 & 1 & 88,9 \\
\hline CCE & 1 & 0 & 1 & 1 & 0 & 1 & 0 & 1 & 0 & 55,6 \\
\hline CMM & 1 & 0 & 1 & 1 & 1 & 0 & 1 & 1 & 1 & 77,8 \\
\hline COD & 1 & 1 & 1 & 1 & 1 & 1 & 1 & 1 & 1 & 100,0 \\
\hline CPE & 1 & 0 & 1 & 1 & 1 & 1 & 0 & 1 & 1 & 77,8 \\
\hline MAR & 1 & 0 & 1 & 1 & 0 & 0 & 0 & 1 & 1 & 55,6 \\
\hline MDB & 1 & 0 & 1 & 1 & 1 & 1 & 1 & 1 & 1 & 88,9 \\
\hline MOD & 1 & 0 & 1 & 1 & 1 & 1 & 0 & 1 & 1 & 77,8 \\
\hline MUC & 1 & 0 & 1 & 1 & 1 & 0 & 0 & 1 & 1 & 66,7 \\
\hline REN & 0 & 1 & 1 & 0 & 1 & 1 & 1 & 1 & 0 & 66,7 \\
\hline UNA & 1 & 0 & 0 & 1 & 1 & 1 & 0 & 1 & 0 & 55,6 \\
\hline UR1 & 1 & 0 & 0 & 1 & 1 & 1 & 1 & 1 & 0 & 66,7 \\
\hline UR2 & 1 & 0 & 0 & 1 & 1 & 1 & 1 & 1 & 0 & 66,7 \\
\hline UR3 & 1 & 0 & 0 & 1 & 1 & 1 & 1 & 1 & 1 & 77,8 \\
\hline UR4 & 1 & 0 & 0 & 1 & 1 & 1 & 1 & 1 & 1 & 77,8 \\
\hline UR5 & 1 & 0 & 0 & 1 & 1 & 1 & 1 & 1 & 0 & 66,7 \\
\hline UR6 & 1 & 0 & 0 & 1 & 1 & 1 & 1 & 1 & 1 & 77,8 \\
\hline UR7 & 1 & 0 & 0 & 1 & 1 & 1 & 1 & 1 & 1 & 77,8 \\
\hline \multicolumn{10}{|c|}{ Institutions de Crédit Direct - ICD } & 86,7 \\
\hline CFAD & 1 & 0 & 1 & 1 & 1 & 1 & 0 & 1 & 0 & 66,7 \\
\hline FINA & 1 & 0 & 1 & 1 & 1 & 1 & 1 & 1 & 1 & 88,9 \\
\hline PAD & 1 & 1 & 1 & 1 & 1 & 1 & 1 & 1 & 1 & 100,0 \\
\hline PAP & 1 & 1 & 1 & 1 & 1 & 1 & 1 & 1 & 0 & 88,9 \\
\hline VIT & 1 & 1 & 1 & 1 & 1 & 1 & 1 & 1 & 0 & 88,9 \\
\hline \multicolumn{10}{|c|}{ Organisations Non Gouvernementales - ONG } & 59,3 \\
\hline ASOP & 1 & 0 & 1 & 1 & 1 & 0 & 0 & 1 & 1 & 66,7 \\
\hline IAM & 0 & 0 & 1 & 0 & 1 & 0 & 1 & 1 & 0 & 44,4 \\
\hline ID & 1 & 0 & 1 & 0 & 1 & 1 & 1 & 1 & 0 & 66,7 \\
\hline \multicolumn{10}{|c|}{ Ensemble de l'échantillon } & 73,3 \\
\hline
\end{tabular}

Source : Données collectées auprès des IMF, février 2007. 